\title{
Family Planning Programs - An Opportunity to Integrate Preconception Health Services into Primary Health Care
}

\author{
Dumitru Siscanu $^{1 *}, \mathrm{PhD}$; Sarah Verbiest ${ }^{2}, \mathrm{DrPH}$; Corina Iliadi-Tulbure ${ }^{3}, \mathrm{PhD}$; \\ Anait Yu. Marianian 4 , PhD, ScD; Patricia Chico Aldama ${ }^{5}$, MPH; Iurii Arian³; Viorica Chihai ${ }^{3}$ \\ ${ }^{1}$ Gheorghe Paladi Public Medical Institution Municipal Clinical Hospital, Chisinau, Republic of Moldova \\ ${ }^{2}$ University of North Carolina at Chapel Hill, School of Medicine, Center for Maternal and Infant Health, \\ Chapel Hill, NC, USA \\ ${ }^{3}$ Nicolae Testemitanu State University of Medicine and Pharmacy, Chisinau, Republic of Moldova \\ ${ }^{4}$ Scientific Centre for Family Health and Human Reproduction Problems, \\ Irkutsk, the Russian Federation \\ ${ }^{5}$ National Institute of Pediatrics, Mexico City, Mexico
}

\begin{abstract}
A life-course approach to enhancing maternal and child health by improving parental health prior to conception is gaining international interest. Healthcare providers and researchers are seeking effective ways to increase women's and men's access to preconception care, especially through primary care. At the same time, preconception health includes conversations about pregnancy planning (or avoidance), which is a part of family planning (FP) services. Unfortunately, "family planning" has the same meaning as "contraception" for many people at the moment, which is too simplistic because there are multiple aspects of preconception health and well-being. Expanding the understanding of preconception health as inclusive of FP and primary care has the potential to increase access to this important care while respecting sexual and reproductive rights, according to a couple's reproductive plans.

Conclusion: Respecting the sexual and reproductive rights of the population requires that FP programs be focused not only on contraception but, equally, on preconception care.(International Journal of Biomedicine. 2021;11(4):467-472.)

Key Words: preconception health $\bullet$ preconception care $\bullet$ family planning $\bullet$ contraception $\bullet$ primary care $\bullet$ reproductive health

For citation: Siscanu D, Verbiest S, Iliadi-Tulbure C, Marianian AYu, Aldama PCh, Arian Iu, Chihai V. Family Planning Programs - An Opportunity to Integrate Preconception Health Services into Primary Health Care. International Journal of Biomedicine. 2021;11(4):467-472. doi:10.21103/Article11(4)_PV
\end{abstract}

\section{Abbreviations}

FP, family planning; GPs, general practitioners; PCHC, preconception health and care; PC, preconception care.

Tmproving the health of the mother and child through programs focused on reducing risks prior to pregnancy in the context of a life-course approach is gaining international interest. Such an evolution in maternal and child health is logical and important. While a focus on

*Corresponding author: Dumitru Siscanu, PhD. Gheorghe Paladi Public Medical Institution Municipal Clinical Hospital, Chisinau, Republic of Moldova. Email:d_siscanu@yahoo.com treating diseases and/or health complications has long been considered a priority in health care, the promotion of simple and cost-effective preventive measures with a positive impact on health are also of importance in medical practice. As such, the recommendation that couples planning a pregnancy should receive care may seem obvious to many medical workers and a considerable part of the population. Thus, discussions about the role of PCHC may seem unnecessary, and the general population may find them simple and unnecessary to address 
in the face of other more important and complicated issues. Preparing couples for a future pregnancy is a since-the-worldbegan topic that is still relevant. Globally, only about half of pregnancies are planned, and the number of couples who have received preconception preparation is much smaller.

A major impediment to progress is the lack of resources required to implement PCHC programs. At the European Congress on Preconception Health (2019), the participants, including those from economically developed countries, appreciated this issue as a very current one. In this context, one strategy recommended by many researchers is the integration of preconception care (PC) services into primary health care. It seems, however, that this process also faces some obstacles: frequently, the motivation of the family doctors/GPs is not enough to achieve the integration in daily practice.

At the same time, preconception preparation requires an interest in and participation of a couple or woman in thinking about pregnancy. Some experts have suggested that interventions to improve reproductive and preconception health could be more relevant and efficient by integrating PCHC services into FP programs, embedded into primary health care. The biggest challenge is that the concept of family planning, with some exceptions, is considered to be synonymous with contraception, i.e., FP $=$ contraception. We propose a different approach: that " $\mathrm{FP}=$ contraception + preconception care." ${ }^{\prime(1,2)}$ There was a surprising amount of resistance by some well-known people in the field of medicine, as well as among researchers and some international organizations, who promote the first approach. It is difficult to explain why preconception healthcare services have been ignored by the same international organizations responsible for developing global strategies and policies to advance family health, which determine health policies and programs at the regional and national levels.

Contraception and PC are two components of FP that interact with each other. Before making the decision to discontinue contraception, the woman or couple should evaluate their preconception risks and have support to reduce them. Access to all contraceptive methods is equally important for couples who do not wish to conceive and those who need to wait until they have been able to address risk factors or built protective factors.

\section{Preconception care}

PC is defined by the WHO as providing biomedical, behavioral, and social health interventions before pregnancy to improve health and modify behaviors and environmental influences. PC can contribute to reducing maternal and childhood mortality and morbidity, and to improving maternal and child health in both high- and low-income countries. ${ }^{(3)}$ Clinicians and health workers should provide the information that a woman and her partner need, in order to make informed choices about planning a pregnancy, to ensure the best possible outcome for the couple and the baby. ${ }^{(4)}$

$\mathrm{PC}$ is an important intervention to reduce risks in early pregnancy and lead to healthy outcomes for women and babies. General PC is directed at all prospective parents and takes place in primary care (GPs or midwife level). Most preconception health messages are important for women and couples regardless of their desire to have a child and include key preventive health measures that should be part of quality, routine care. PC includes risk assessment, health promotion, counseling and, if indicated, referral to a specialist to assist with unique needs (e.g., a nutritionist, an endocrinologist). Although the primary goal is to promote better reproductive outcomes, the changes induced by PC are also usually beneficial to the woman's health. Furthermore, PC aims to improve couples' informed decision making by providing information on reproductive options. ${ }^{(5)}$ One size cannot fit all, however. The content and mechanisms of delivery of preconception will need to be tailored to the realities of different countries. Even where strong public health programs are in place across the life-course, they do not guarantee that women enter pregnancy in good health. ${ }^{(6)}$ The concept of preconception health care was described almost two centuries ago when it was suggested that the medical treatment of children should begin with the earliest formation of the embryo and should include the mother before marriage as well as during pregnancy. Many years later, PC has not become part of the routine care of most reproductiveaged women. ${ }^{(7)}$

The paradox of the situation is that while more researchers have shown the important role of PC for the health of generations at all stages of life, the practical implementation of the recommendations of improving the preconception health remains suboptimal, with considerable differences between countries. It seems that the role of PC of couples in the prevention of maternal-fetal complications, both at the antenatal and postnatal stage, has become, over time, a declarative statement. Even in the countries with welldeveloped health management systems, frequently, program managers give priority to strategies with more immediate impact compared to interventions that will deliver results in the medium or long term. This is largely due to the social policies of the governments of many countries, especially in the context of limited budgets, which give priority to projects that have short-term outcomes. Thus, it is "more convenient" to purchase some modern ultrasonography devices to detect congenital defects or to strengthen the intensive care units for infants with incubators, than invest in the health of young adults for several years before they intend to start a family. While investment in any and all interventions aimed at improving the health of the mother and child are welcome, policies and programs need to include preventive strategies that ultimately can help sustain ongoing good outcomes for families. For example, ignoring the $\mathrm{PC}$ stage will still maintain the high frequency of congenital malformations in fetuses and newborns, and the constant number of preterm births will require increased effort from intensive therapy units for premature children, sophisticated medical equipment, expensive drugs, etc., as well as the economic, social and psychological costs, both at the family and community level.

Providing quality PC is the responsibility of all primary care providers, not just those who provide maternity care or handle a high volume of women's preventive health visits. Innovative strategies that incorporate PC into routine primary care visits are needed ${ }^{(8)}$ Transforming the way PC is delivered is critical to success. In order to successfully deliver PC, family 
physicians must understand the risk factors for- and the realities of- unintended pregnancy; recognize the value of reproductive planning in reducing these risks, and assess preconception health risks during chronic disease management visits and acute care visits that are not specifically focused on women's health or maternity issues. Preconception care is primary care and it should be a priority for primary care providers in all settings. The majority of preconception health topics are important whether a woman desires a future pregnancy or not, so providing quality PC is essentially providing quality women's health care.

At the same time, doctors across specialties should also consider the possibility of pregnancy development in reproductive age women and they should inform them about possible risks for their health, for fetal and newborn risks, generated by disease evolution and treatment. ${ }^{(9)}$

Integrating preconception planning services into primary health care is important to ensure that services reach larger numbers of people in the reproductive age population and that people receive quality counseling and services from providers who know them. ${ }^{(10)}$ In 2006, the Center for Disease Control (CDC) released Recommendations to Improve Preconception Health and Health Care - United States: A Report of the CDC/ ATSDR Preconception Care Work Group and the Select Panel on Preconception Care. This report was published in an effort to improve reproductive health outcomes. However, in spite of these national and international efforts, there continue to be barriers to preconception counseling in routine primary care.

Steel et al. ${ }^{(1)}$ conducted a systematic review of barriers in the process of providing preconception services and programs. The most consistently cited barrier for PC provision, as described by primary care practitioners and specialist (obstetrician-gynecologist) doctors are insufficient clinical time and a lack of reimbursement for practitioner time spent on preconception counseling. Primary care practitioners participating in a United Kingdom survey asserted that a potential barrier to the success of PC programs is the perceived low value placed on PC held by women in the community. These health professionals purported that PC programs do not reach those women who do not plan their pregnancy. To add complexity to this issue, women's lack of knowledge about PC has also been highlighted by both Dutch midwives and Israeli obstetrician-gynecologists as a potential barrier to effective care delivery as the initial clinical encounter for many women often occurs in the early antenatal period. Conversely, a concern has been expressed by primary care practitioners that providing information on the teratogenic effects of medication within preconception counseling will lead to women avoiding important pharmaceutical care. This was of particular concern if the practitioner was not confident in identifying a woman's pregnancy plans during the clinical encounter.

Primary care providers also face difficulties with prioritizing PC together with other preventive care issues. Consequently, potential interventions for improving the delivery of PC guidelines should also focus on providing tools and resources to assist providers in delivering the content and evidence base of the guidelines. Understanding the views of both women and providers as well as the theoretical basis for changing their behavior will be essential when designing effective implementation strategies for improving the delivery and uptake of PC. ${ }^{(12)}$ These strategies may also need to consider the role practice nurses and other health professionals may have in facilitating better uptake of PC, especially among high-risk patients who should need services the most.

A review by Shannon et al. ${ }^{(13)}$ identified primary care as the most common setting for preconception health service. However, the authors also concluded that there is no agreed consensus on the best method to deliver care within primary care. It is possible that many strategies acting synergistically are needed to improve service delivery. Integration into routine care could be one strategy, but this would not be sufficient to reach the target population for routine preventive care as seen in some other countries. In another aspect, Ukoha and Dube ${ }^{(14)}$ reported that although preconception care is recognized as an important factor in improving pregnancy outcomes, most primary healthcare nurses lack the necessary resources to provide PC. Given that the majority of preconception health messages are also key preventive health messages for women, there may either be confusion in terms of what PC counseling entails or providers may be providing suboptimal guidance and support for women's health overall. For example, all people should be screened for tobacco use and given guidance if they screen positive for use. This should be done regardless of a person's gender or age, and tobacco use has negative consequences. For a couple expressing an interest in pregnancy or struggling with fertility, preconception health messages may be an extra incentive for seeking support in tobacco cessation.

Pre-pregnancy preparing as a component of FP services

The shift to integrate preconception health promotion into the continuum of women's health care requires a diverse multi-level and multi-strategic approach involving a range of sectors and health professionals to improve access to care and address the determinants of health. ${ }^{(15)}$ Considering multiple problems in the provision of preconceptional or interconceptional health services, especially the lack of resources for providers, on the one hand, and the modest motivation of the population to benefit from these services, on the other, managers in the field tried to integrate PC into FP programs. There are not many published data on this subject.

Hussein ${ }^{(16)}$ mentioned that since the general practitioners' primary concern is that it is difficult to convince women to have a preconception assessment, it would seem feasible to reach them through FP clinics, which have the potential to be a good setting to deliver preconception assessment of reproductive risks.

Following the Population and Development Conference in Cairo in 1994, in many countries national programs were developed and implemented to offer FP services by opening specialized clinics and providing equipment and training for service providers. FP services were included in the basic packages of primary health services. The state programs in the field of FP encouraged cross-sector collaboration across the public systems of health, education, social assistance, etc. National health systems have also ensured the interdisciplinary cooperation of the medical providers of different specialties in promoting and providing FP services. 
Preconception health messages, recommendations and guidelines originated in the USA and the preconception movement has gained momentum internationally with a variety of strategies developed and tested for improving preconception health and related outcomes. ${ }^{(10,17)}$ The report titled Providing Quality Family Planning Services: Recommendations of CDC and the US Office of Population Affairs (2014) provides strategies developed collaboratively by the CDC and the Office of Population Affairs of the US Department of Health and Human Services. These agencies advance recommendations for the provision of quality FP services, which include contraceptive services, pregnancy testing and counseling, helping clients achieve pregnancy, basic infertility services, preconception health services, and sexually transmitted disease services. Gavin et al. ${ }^{(18)}$ conclude that providers of FP services should offer preconception health services to female and male clients in accordance with $\mathrm{CDC}$ recommendations to improve preconception health and health care. Including PC in FP services could ensure a higher quality of couple preparation for pregnancy, including more active involvement of men.

Preconception preparing could be used more fully in cases of infertility, which is one of the FP important areas. According to the Royal College of Obstetricians and Gynecologists (2011), the lack of preconception care for infertile couples may be a reflection of a lack of continuity in women's health reported elsewhere. ${ }^{(19)} \mathrm{PC}$ should be actively offered by all healthcare professionals. Nurses in infertility clinics and GPs in primary care are ideally placed to open up a discussion about PC with couples seeking fertility assistance, promoting the agency of these patients during investigation and treatment stages.

Another category of target beneficiaries of FP services are adolescents. It is crucial to create a responsible attitude for future decisions regarding the conception of a child, in adolescents and young adults. The concept of maintaining/ fortifying health in general, as well as risk-free behavior, should be presented to young people as a condition of paramount importance for achieving their reproductive plans.

However, there are some controversies about this approach. In 2018, the WHO published "Family Planning. A Global Handbook for Providers (3rd edition)," which is considered to be a key document that contributes to ensuring the quality and safety of FP services, encouraging all national health systems and other interested organizations to engage in this important topic. ${ }^{(20)}$ This document includes a very modest section regarding $\mathrm{PC}$, the other part of the document being devoted to contraception. The information provided on the subject is connected by the fact that a woman who wants to have a baby can use advice on preparing for pregnancy and giving birth safely, thus having a healthy child. There are some very general recommendations for women only, including supplementation with folic acid and iron. This manual, along with other multiple sources, confirms that in principle, FP is meant only to prevent pregnancy; in other words, "FP = Contraception." The term "family planning" is often considered by the general population and healthcare providers as synonymous with contraception.
Regarding the subject of FP and respect for human rights, the document "Human Rights: Family Planning Providers' Contribution" mentions the following: "All people deserve the right to determine, as best they can, the course of their own lives. Whether and when to have children, how many, and with whom are important parts of this right. FP providers have the privilege and responsibility to help people to make and carry out these decisions. Furthermore, programs that honor their clients' human rights contribute to positive sexual health outcomes."(21) And yet, the manual titled "Family Planning A global handbook for providers," only provides information about contraception for healthcare professionals in low- and middle-income countries. This approach can raise several questions, one of which could be: What is the interpretation (meaning) of the concept of FP for health providers in highincome countries? We believe it is unfair that the promotion and respect of people's sexual-reproductive rights depend on the economic status of the countries.

The results of PerConcept survey (2019), conducted among family physicians and other specialists in three countries: Republic of Moldova (Chisinau), Mexico (Mexico City), and the Russian Federation (Irkutsk), confirmed that the concept of family planning should really include the component of preconception preparation, along with contraception, the approaches being applied equally to both. The results of the survey are interesting because doctors, respondents from three regions of the world: Europe, Asia, and North America, have similar perceptions about including PC in FP services. ${ }^{(22)}$ The majority of respondents considered that strategies and programs in the field of FP should be revised to include preconception health services. In the practical aspect, the interviewees mentioned the importance of ensuring that the population has access to information and services in reproductive health, including PC, as "an effective tool to prevent some risks in maternal and infant well-being."

\section{The interaction between two componentsof FP:} contraception and PC

There is an important connection between pregnancy prevention and pregnancy preparing. In some cases, PC requires the use of a safe contraception method until the couple is able to address health and behavioral problems to minimize the risks to a healthy pregnancy. At the same time, a couple's decision to stop using contraception in order to become pregnant offers a "teachable moment" to provide preconception preparation information. Frayne ${ }^{(23)}$ suggests that providers can reach women who are planning a pregnancy with preconception health messages at scheduled contraception visits or annual wellness examinations. van der Zee et al. ${ }^{(5)}$ consider that an important requirement for preconception consultation is pregnancy planning, which is possible through the provision of contraceptives. Incorporating pregnancy intention screening into primary care to address unmet preconception and contraception needs may improve the delivery of FP services and improve reproductive health and wellness overall. ${ }^{(24)}$

In the position paper on Preconception Care of the American Academy of Family Physicians, one of the General Recommendations for Preconception Interventions for Women and Men is contraception. ${ }^{(10)}$ This position paper emphasizes 
the importance of incorporating routine identification of pregnancy intention for men and women of reproductive age to inform shared decision-making around reproductive planning and risk reduction. Because reproductive choices and risks can change over time, preconception health risks and pregnancy desires should be routinely assessed during chronic disease management visits.

Women recognize that pregnancy planning is an important benefit of contraception; however, they recognize it as a disadvantage also, as it is difficult to decide when to stop its use and to try to become pregnant. ${ }^{(5)}$ One of the determining causes of the situation described could be the lack of information on fertility, menstrual cycle, and fertile days, which are important components of PC. In another context, it should be realized that the values and ideas of "naturalness" and "romanticism" that people consider important in the process of becoming pregnant. In other cases, women (or men) may feel coerced into pregnancy, and in others, people may feel overall that they have limited control of their lives and therefore limited capacity to plan for the future. Due to this complexity, confidentiality is essential for people who ask medical workers for PC. . $^{(5,25)}$

Primary care for women of childbearing age should include routine assessment of a woman's reproductive goals and pregnancy intentions ("reproductive planning"). ${ }^{(26,27)}$ Women who could potentially become pregnant should be assessed for preconception risks and educated about the importance of maternal health in ensuring healthy pregnancies. Women may be motivated to address modifiable health risks by learning about the way their health will affect a future pregnancy. For women not intending to get pregnant in the short term, PC should include counseling on effective contraception. Women with chronic medical conditions should be counseled about highly effective reversible methods, such as intrauterine devices and contraceptive implants, which have few medical contraindications. Including preconception health services in FP care could provide more comprehensive care to meet the unique needs of all beneficiaries and will be more appropriately accepted by women and men. There are a number of key reports and recommendations from the $\mathrm{CDC}(2006,2010)$ and $\mathrm{WHO}(2013)$ that encourage providing comprehensive services to people of reproductive age.

The strategies and resources used to facilitate the clinical and public health integration and dissemination of PC, including the role of group care, the medical home, workplace and school-based health promotion programs, and home visitation, will also impact future progress. ${ }^{(28)} \mathrm{We}$ also recognize that there are many factors that are out of the individual's control that can influence their fertility, health and well-being including environmental hazards in their work place and community, inadequate access to health care and follow-up resources, inadequate access to healthy food and places to exercise, and stress due to poverty and racism or bias. Telling people to change something that is not in their control can be counterproductive. Therefore, it is important that providers and clinics partner with public health professionals, community leaders, and policymakers to create healthy conditions where all people can survive and thrive.

\section{Conclusions}

Modifying FP strategies and programs to include the preconception component, will contribute to:

- opening up optimistic prospects for integrating PC services into primary care, as FP programs have been implemented in many countries, involving financing operations and the infrastructure services system, in particular with the involvement of family doctors/GPs;

- respect for the sexual and reproductive rights of all persons and couples, regardless of their reproductive plans;

- increased opportunities for providing health education and information about PC. Training in pregnancy preparing could be carried out in FP networks (specialized clinics and health centers for young people, medical and private institutions in the field of reproductive health, etc.) for medical care providers in primary care and women and men at the same time;

- continuity of reproductive health care;

- better sustainability of maternal and child health programs and projects, taking into account the longer-term benefits of FP activities, including PC;

- last but not least, increase the value of FP services for the population, as they will increase their impact on maternal and child health indicators.

Approaching PC as a component and integral part of FP can generate discussions between providers or researchers, and future high-quality studies will be needed to adjust public health policies and improve clinical practices.

\section{Acknowledgments}

This article contains material that has been discussed at the VIII International Research and Practical Conference «FUNDAMENTAL AND APPLIED ASPECTS OF REPRODUCTION» (December 2021, Irkutsk, Russia). The author thanks all researchers who participated in the oral discussion.

\section{Conflict of Interests}

The authors declare that they have no conflicts of interest.

\section{References}

1. Shishcanu D, Marianian AY, Iliadi-Tulbure C. Preconceptional care: opportunities and challenges. Acta Biomedica Scientifica. 2018;3(3):69-74.

2. Siscanu D, Iliadi-Tulbure C, Bolun A, Chihai V, Gurau V. Suggestions about population perception on preconceptional care in Republic of Moldova. Buletin de Perinatologie. 2018;3(79):91-94.

3. Meeting to Develop a Global Consensus on Preconception Care to Reduce Maternal and Childhood Mortality and Morbidity. World Health Organization Headquarters, Geneva 6-7 February 2012; 67p. Meeting report.

Available from: https://apps.who.int/iris/handle/10665/78067

4. Pre-conception - advice and management. Clinical 
Knowledge Summaries. 2017. Available from: https://www. nice.org.uk/.

5. van der Zee B, de Beaufort ID, Steegers EA, Denktas S. Perceptions of preconception counselling among women planning a pregnancy: a qualitative study. Fam Pract. 2013 Jun;30(3):341-6. doi: 10.1093/fampra/cms074.

6. Preconception care: Maximizing the gains for maternal and child health. World Health Organization. Geneva. 2013. Available from: http://www.who.int/maternal child adolescent/documents/preconception_care_policy_brief.pdf.

7. Lammers Cr. A New Focus on Preconception Healthcare and the Life Course Theory of Health. Journal of Community and Public Health Nursing. 2018;4(2):216. doi: 10.4172/24719846.1000216.

8. van Voorst $\mathrm{S}$, Plasschaert $\mathrm{S}$, de Jong-Potjer L, Steegers E, Denktaş S. Current practice of preconception care by primary caregivers in the Netherlands. Eur J Contracept Reprod Health Care. 2016 Jun;21(3):251-8. doi: 10.3109/13625187.2016.1154524.

9. Pustotina OA. [Preconception Preparation]. Meditsinskiy Sovet=Medical Council. 2017;13:64-70. d o i : 10.21518/2079-701X-2017-13-64-70. [Article in Russian]. 10. American Academy of Family Physicians. Preconception care (position paper). 2016. Available from: https://www.aafp. org/about/policies/all/preconception-care.html

11. Steel A, Lucke J, Reid R, Adams J. A systematic review of women's and health professional's attitudes and experience of preconception care service delivery. Fam Pract. 2016 Dec;33(6):588-595. doi: 10.1093/fampra/cmw094.

12. Mazza D, Chapman A, Michie S. Barriers to the implementation of preconception care guidelines as perceived by general practitioners: a qualitative study. BMC Health Serv Res. 2013 Jan 31;13:36. doi: 10.1186/1472-6963-13-36

13. Shannon GD, Alberg C, Nacul L, Pashayan N. Preconception healthcare delivery at a population level: construction of public health models of preconception care. Matern Child Health J. 2014 Aug;18(6):1512-31. doi: 10.1007/ s10995-013-1393-8.

14. Ukoha WC, Dube M. Primary health care nursing students' knowledge of and attitude towards the provision of preconception care in KwaZulu-Natal. Afr J Prim Health Care Fam Med. 2019 Nov 12;11(1):e1-e8. doi: 10.4102/phcfm. v11i1.1916.

15. Lang AY, Boyle JA, Fitzgerald GL, Teede H, Mazza D, Moran LJ, Harrison C. Optimizing preconception health in women of reproductive age. Minerva Ginecol. 2018 Feb;70(1):99-119. doi: 10.23736/S0026-4784.17.04140-5.

16. Hussein N. Preconception assessment of reproductive genetic risk in primary care. Thesis submitted to the University of Nottingham for the degree of Doctor of Philosophy. December 2016. Available from: http://eprints.nottingham.ac.uk/37967/2/Phd\%20thesis.pdf. 17. Floyd RL, Johnson KA, Owens JR, Verbiest S, Moore CA, Boyle C. A national action plan for promoting preconception health and health care in the United States (2012-2014). J Womens Health (Larchmt). 2013 Oct;22(10):797-802. doi: 10.1089/jwh.2013.4505.

18. Gavin L, Moskosky S, Carter M, Curtis K, Glass E, Godfrey E, Marcell A, Mautone-Smith N, Pazol K, Tepper N, Zapata L; Centers for Disease Control and Prevention (CDC). Providing quality family planning services: Recommendations of CDC and the U.S. Office of Population Affairs. MMWR Recomm Rep. 2014 Apr 25;63(RR-04):1-54.

19. Allan HT, Mounce G, Crespo E, Shawe J. Preconception care for infertile couples: Nurses' and midwives' roles in promoting better maternal and birth outcomes. J Clin Nurs. 2018 Dec;27(23-24):4411-4418. doi: 10.1111/jocn.14586.

20. Family Planning. A global handbook for providers. Evidence-based guidance developed through worldwide collaboration, 3rd edition, 2018. Available from: https:/apps. who.int/iris/bitstream/handle/10665/260156/9780999203705eng.pdf;jsessionid=027939A21D681CBFFC67C66E0DBB28 FD? sequence $=1$

21. Ensuring human rights in the provision of contraceptive information and services. Guidance and recommendations. WHO. 2014. Available from: https://www.who.int/ reproductivehealth/publications/family_planning/humanrights-contraception/en/.

22. Siscanu D, Iliadi-Tulbure C, Marianian AY, Aldama PC, Verbiest S, Dumbraveanu I, et al. "PerConcept" Study: Provider Opinions about Integrating Preconception Care into Family Planning Services. Public Health, Economy and Management in Medicine, 2021;91(4).

23. Frayne DJ. Preconception Care Is Primary Care: A Call to Action. Am Fam Physician. 2017 Oct 15;96(8):492-494.

24. Srinivasulu S, Falletta KA, Bermudez D, Almonte Y, Baum R, Coriano M, Grosso A, Iglehart K, Mota C, Rodriguez L, Taveras J, Tobier N, Garbers SV. Primary care providers' responses to pregnancy intention screening challenges: community-based participatory research at an urban community health centre. Fam Pract. 2019 Nov 18;36(6):797803. doi: 10.1093/fampra/cmz027.

25. Bortolus R, Oprandi NC, Rech Morassutti F, Marchetto L, Filippini F, Agricola E, Tozzi AE, Castellani C, Lalatta F, Rusticali B, Mastroiacovo P. Why women do not ask for information on preconception health? A qualitative study. BMC Pregnancy Childbirth. 2017 Jan 5;17(1):5. doi: 10.1186/ s12884-016-1198-z.

26. Callegari LS, Ma EW, Schwarz EB. Preconception care and reproductive planning in primary care. Med Clin North Am. 2015 May;99(3):663-82. doi: 10.1016/j.mcna.2015.01.014. 27. Dean SV, Lassi ZS, Imam AM, Bhutta ZA. Preconception care: promoting reproductive planning. Reprod Health. 2014 Sep 26;11 Suppl 3(Suppl 3):S2. doi: 10.1186/1742-4755-11-S3-S2.

28. St Fleur M, Damus K, Jack B. The future of preconception care in the United States: multigenerational impact on reproductive outcomes. Ups J Med Sci. 2016 Nov;121(4):211215. doi: 10.1080/03009734.2016.1206152. 